\title{
Designing the Smart Badge: A Wearable Device for Hospital Workers
}

\author{
Ricardo Peixoto \\ Fraunhofer Portugal AICOS \\ Porto, Portugal \\ ricardo.peixoto@fraunhofer.pt
}

\author{
Jorge Ribeiro \\ Fraunhofer Portugal AICOS \\ Porto, Portugal \\ jorge.ribeiro@fraunhofer.pt
}

\author{
Eduardo Pereira \\ Fraunhofer Portugal AICOS \\ Porto, Portugal \\ eduardo.pereira@fraunhofer.pt
}

\author{
Francisco Nunes \\ Fraunhofer Portugal AICOS \\ Porto, Portugal \\ francisco.nunes@fraunhofer.pt
}

\author{
Ana Pereira \\ Fraunhofer Portugal AICOS \\ Porto, Portugal \\ ana.pereira@fraunhofer.pt
}

\begin{abstract}
Wearable self-monitoring technologies can help users learn more about themselves. By enabling people to track their habits and visualising them, wearables can support selfawareness and self-care. For example, wearables can help shift workers dealing with unorthodox sleep schedules of shift work through tools that monitor their body and habits. However, current wearable technologies do not fit well in healthcare scenarios where workers engage in specific antiseptic practices. In this paper we present the process of designing the Smart Badge, a wearable for monitoring physical activity, sleep, and light exposure of hospital workers.
\end{abstract}

\section{CCS CONCEPTS}

- Applied computing $\rightarrow$ Health informatics; • Humancentered computing $\rightarrow$ Mobile devices;

\section{KEYWORDS}

Activity Monitoring, wearable sensors, self-monitoring, rapid prototyping, healthcare workers, shift work

\section{INTRODUCTION}

Recent years have seen an expansion of wearables that can be worn during daily activities $[2,10]$. Wearables are often used for tracking chronic conditions and overall wellbeing $[9,15]$, but more recent work has started tracking work related activities as well $[3,5,13]$.

Nevertheless, monitoring the activities of hospital workers is not an easy task. Antiseptic practices prevent doctors, nurses, and nurse assistants from wearing things in their wrists, restricting the use of commercial activity monitors, such as Fitbit Flex 2 or Apple Watch. The exception is the badge that many workers wear on the neck, used to access different rooms and display their identification.

This study documents the design of the Smart Badge, a wearable device for monitoring physical activity intensity, sleep, and light exposure of hospital workers, that fits antiseptic practices. We start by briefly describing insights from the interviews with hospital workers used to define the system requirements. Then we detail the design process of the Smart Badge, by showing different versions of the artifact.

The contribution of this paper lies in the description of the Smart Badge, a self-monitoring wearable for shift workers who work in the hospital. The design process also contributes to the research community, by documenting how needs and practices of shift workers could be addressed with a technological solution.

\section{BACKGROUND}

People have a natural tendency to observe and reflect about their body and lives [9, 10]. Surveys consistently demonstrate that people track their physical activity, even though sometimes only on their head [4]. To help people track their physical activity, companies developed wearable sensors with algorithms that count steps, classify activities, and estimate distance. The purpose of tracking physical activity also expanded from fitness [14] and sleep [2], to work [5]. 

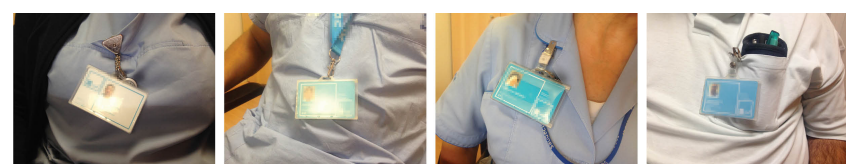

Figure 1: Current identification badge of hospital workers interviewed, being used in different positions.

Tracking work activities is not a new topic. After all Ergonomics and Human Factors have been devoted to the issue for decades. Still most studies followed an organizational perspective, focusing on monitoring of activities out of the office [5], tracking work-breaks during the day [3], social interactions [12], number of working hours and it's correlation with errors in an clinical working context $[17,18]$.

Monitoring the activities in healthcare scenarios is not easy though. The majority of commercial solutions use the wristband form factor [8], which goes against antiseptic medical practices. Recent research discusses the use of clothes with sensors [6] or tattooed jewelry [7], but these solutions seem experimental at this point.

As appropriate solutions for tracking shift workers in the healthcare setting were lacking, we decided to design a new wearable.

\section{METHODS}

We started the project with semi-structured interviews with hospital workers. These interviews aimed to understand the work context of hospital workers and explore the issues faced by such professionals during shift work. A total of 26 workers (13 medical assistants, 9 nurses, 2 doctors, and 2 security officers) participated in the study. 20 participants were over 45 , and 6 were on their late twenties or early thirties. All professionals worked in shifts, and all did at least one night shift per week.

Interviewees were recruited through personal contacts of the research team and through a large local healthcare provider. Two or three researchers were present during the interviews and each interview took approximately $30 \mathrm{~min}$ utes. Participants provided informed consent after hearing an explanation about the project and the goals of the interviews. The conversations were audio recorded, transcribed verbatim, and coded for themes with thematic analysis [1].

The interview script focused on the issues, challenges, and characteristics of shift work. It also explored the concerns regarding the usage of the badge in a hospital environment.

The design of the Smart Badge followed a Research Through Design approach [19] to learn from the artifact as the design process unfolded. Consequently, several physical prototypes were created during the process.

\section{USER RESEARCH AND CASING DESIGN}

Throughout the interviews participants mentioned their strategies for balancing work, rest, and daily life, and the importance of making sure they were having enough sleep, despite their erratic schedule. Monitoring physical activity and sleep periods helped workers understand if they were resting enough; and monitoring light exposure, indicated if workers were exposed to enough light during their shifts. Moreover, the interviews documented the usage of digital devices and jewelery on an hospital environment.

Using the smartphone as a sensor for monitoring the worker would probably be the most elegant solution; however, this solution would require hospital workers to carry out their personal smartphone at all times during work shifts. In addition, the assessment of light exposure would not be possible, since the smartphone is sometimes carried inside the pocket. Another prominent idea was to design a device to wear on the wrist, similar to most activity trackers; however, medical practices and hospital policies discourage healthcare workers from wearing anything on their wrists and hands. Jewelery pieces such as watches, bracelets, rings, necklaces, or even earrings are also banned or at least strongly discouraged. The only exception was the identification badge (Figure 1), which was used to give access to reserved areas of the hospital and/or display one's identification.

Fitting sensors inside the identification badge has some advantages for the hospital setting. Workers can wear a monitoring device without breaching antiseptic restrictions, as the badge is already an exception. There is no risk of the workers standing out with the badge, because it seems like a normal badge and easily blends into their uniform. Moreover, integrating the sensors into the badge leverages on existing habits, thus it is less one object to forget to carry.

After settling with the badge as the casing for the sensors, we wanted to understand where workers used it, given the influence of the badge position on the measuring of physical activity and light exposure. Workers needed to have the identification badge visible at all times during work. However, workers were free to place the badge anywhere they wanted as long as it was visible. According to the photographic records (Figure 1) and the insights from interviews, workers would either use the badge around the neck or clipped to the uniform. Some interviewees reported to prefer to fix the badge to the uniform because when the badge was worn around the neck attached to a ribbon, it could hit the patient when performing some activities. Workers used a spring clip to attach the badge to the uniform, and the badge was usually worn around the upper torso. 


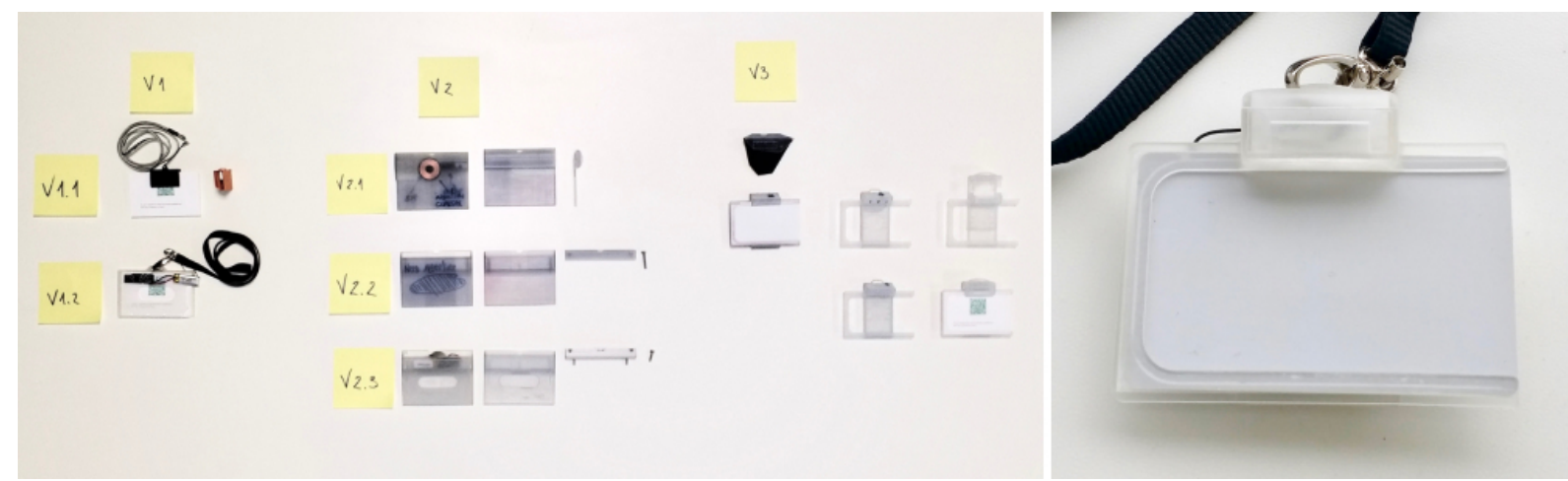

Figure 2: Previous iterations of the badge casing (left). Latest version (right).

\section{Physical prototyping}

Following the first insights from the interviews, we created two conceptual versions of the Smart Badge: v1.1 and v1.2. The idea was to open the design space to different solutions. Smart Badge v1.1 used a minimalist approach that would give further visibility to the card, while Smart Badge v1.2 was meant to be very similar to existing badges and hold the electronic components inside (see Figure 2). However, when reflecting about the versions created, we intuited that using a minimalistic design would be detrimental as the card could easily fall and raise attention to the device. Version 1.2 follows a more conservative approach by exploiting the classic form factor of an identification badge. Despite the less creative approach, it blended better with the worker's uniform. Given these considerations we decided to evolve the prototype with version 1.2, rather than 1.1.

The Smart Badge v2.1 evolved to further resemble a common badge, ensuring it would blend well with the healthcare setting. We 3D printed the case, placed the electronics, sealed the badge, and created a way to open the Smart Badge for maintenance. After using version 2.1, we found three issues affecting the usability: a) The nonexistence of a feature to pull the card out; b) Cover was too thin, and would easily break; and c) Bulky dimensions.

In version 2.2 we changed the opening from the side to the top to make the cover stronger. The case became slimmer and more elegant; still the top cover was too wide and suitable to let liquids in during cleaning. V2.3 was very similar to v2.2, but had a front opening for removing the card.

Version 2.3 was discussed with nurses and medical assistants at the hospital. Participants considered this version comfortable to use, appealing and with the appropriate weight for moving constantly.

In version 3 we focused on reducing the material necessary to make the object to a minimum at the same time that we started to refine the placement of the electronic components. The components consist on a custom PCB with $32 \times 13 \times 4 \mathrm{~mm}$, a lithium battery, and an induction coil with a diameter of $22 \mathrm{~mm}$ and a thickness of $0.8 \mathrm{~mm}$. The induction charging feature is of paramount importance to avoid apertures on the device, which allows the proper sanitation often required. This version also includes a reinforced stripe hold area and a cut out side for the identification card.

There are two advantages in using the badge form factor. On one hand, the possibility of people forgetting to use an external device was eliminated, as they already need to wear the badge for security clearance. One advantage of using the badge form was that it was it was possible to use wearable sensors without going against current rules and regulations of the hospital. The main goal was to develop a device that would blend in with the environment, without disturbing patients and other hospital workers. In other words, the proposed design could be mistaken for a standard badge. This principle aligns with Mann's advice for designing wearable devices, which argues that the visibility of certain technologies raises issues in social interactions with others [11].

\section{SMARTPHONE APPLICATION}

The Smart Badge contains an accelerometer that implements a machine learning algorithm trained to classify physical activity intensity into four different levels: sedentary, light, moderate, and vigorous [16]. To make sense of these personal and environmental data, we developed an Android application that connects with the Smart Badge. We are currently exploring how to visualize physical activity data in a manner that can help workers to self-reflect about their effort during work activities. The current solution (Figure 3) depicts an initial step towards that goal. With the current proposal, workers are capable of visualizing three metrics: sleep, activity, and light exposure. For each one, workers are able to check data for the current shift, as well as past data.

Regarding the 'Activity' user interface, the bar shows 4 colors representing the percentage of time workers have spent on each activity intensity level (sedentary, light, moderate, 
and vigorous), giving a broad perspective of the physical effort invested during the current or past shift. This information will enable hospital workers to reflect on their practices and enable them to make decisions regarding their physical health and well-being.

\section{DISCUSSION}

This study described the design of a wearable device to monitor physical activity and light exposure of hospital workers. To our best knowledge this is the first study to describe a wearable badge, specifically designed for hospital shift workers. While other studies investigated the use of wearables in the workplace $[3,5]$, they did not focus on enabling shift workers to self-reflect on their habits, nor were they specifically designed for this audience [12].

Designing a device for hospital workers was especially important because, according to our user research, these users adhere to strict antiseptic practices, due to medical and hospital policies. Our solution fits their needs and practices enabling the Smart Badge to exist in their work environment.

Future work will focus on continue developing the smartphone application, as well as piloting the system. The design of the case might also go through some improvements based on user feedback. Moreover, we will be conducting nonsupervised trials with end-users from 3 different countries.

\section{ACKNOWLEDGMENTS}

We acknowledge the shift workers who participated in the project. This work was performed in the context of the

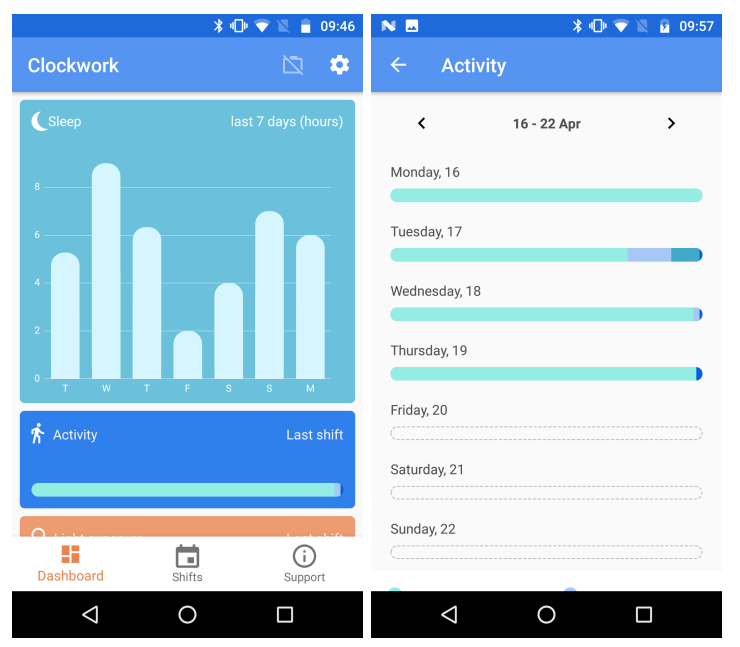

Figure 3: The picture on the left shows the dashboard of the smartphone app. It displays the physical activity and light exposure during a shift, and sleep data from the past 7 days. The picture on the right represents the activity levels achieved during each of the 7 days of a week. project Clockwork (no AAL-2013-6-055) funded under the AAL JP, by the European Commission and National Funding Authorities of Portugal, Hungary, Italy, and Spain.

\section{REFERENCES}

[1] V. Braun and V. Clarke. 2006. Using Thematic Analysis in Psychology. 3, 2 (2006), 77-101.

[2] E. Choe, B. Lee, M. Kay, W. Pratt, and J. Kientz. [n. d.]. SleepTight: low-burden, self-monitoring technology for capturing and reflecting on sleep behaviors. In Proc. of UbiComp '15.

[3] D. Epstein, D. Avrahami, and J. Biehl. [n. d.]. Taking 5: Work-Breaks, Productivity, and Opportunities for Personal Informatics for Knowledge Workers. In Proc. of CHI'16.

[4] S. Fox and M. Duggan. 2013. Tracking for health. Pew Research Center's Internet \& American Life Project.

[5] N. Gorm and I. Shklovski. 2016. Sharing Steps in the Workplace: Changing Privacy Concerns Over Time. In Proc. of CHI'16. 4315-19.

[6] C. Kao, D. Ajilo, O. Anilionyte, A. Dementyev, I. Choi, S. Follmer, and C. Schmandt. 2017. Exploring Interactions and Perceptions of Kinetic Wearables. In Proc. of DIS '17. 391-6.

[7] C. Kao, C. Holz, A. Roseway, and A. Calvo C. Schmandt. 2016. DuoSkin: Rapidly Prototyping On-skin User Interfaces Using Skin-friendly Materials. In Proc. of ISWC '16. ACM, 16-23.

[8] J. Lee and J. Finkelstein. 2015. Consumer Sleep Tracking Devices: a critical review. Digital Healthcare Empowering Europeans: Proceedings of MIE2015 210 (2015), 458-60.

[9] I. Li, J. Froehlich, J. Larsen, C. Grevet, and E. Ramirez. 2013. Personal Informatics in the Wild: Hacking Habits for Health \& Happiness. In Proc. CHI EA '13. ACM, 3179-82.

[10] I. Li, Y. Medynskiy, J. Froehlich, and J. Larsen. 2012. Personal informatics in practice: improving quality of life through data. In Proc. CHI EA '12. ACM, 2799-802.

[11] S. Mann. 1997. Wearable computing: a first step toward personal imaging. Computer 30, 2 (1997), 25-32.

[12] A. Matic, V. Osmani, and O. Mayora-Ibarra. 2014. Mobile Monitoring of Formal and Informal Social Interactions at Workplace. In Proc. of UbiComp '14 Adjunct. 1035-44.

[13] A. Meyer, G.Murphy, T. Zimmermann, and T. Fritz. 2017. Design Recommendations for Self-Monitoring in the Workplace: Studies in Software Development. Proc. of the ACM on Human-Computer Interaction 1, 2 (November 2017).

[14] Dan Morris, T Scott Saponas, Andrew Guillory, and Ilya Kelner. 2014. RecoFit: using a wearable sensor to find, recognize, and count repetitive exercises. In Proceedings of the SIGCHI Conference on Human Factors in Computing Systems. ACM, 3225-3234.

[15] F. Nunes, N. Verdezoto, G. Fitzpatrick, M. Kyng, E. Grönvall, and C. Storni. 2015. Self-Care Technologies in HCI: Trends, Tensions, and Opportunities. ACM Trans. Comput.-Hum. Interact. 22, 6 (2015), 33:133:45.

[16] A. Pereira and F. Nunes. 2018. Physical Activity Intensity Monitoring of Hospital Workers using a Wearable Sensor. In Proc. of PervasiveHealth '18.

[17] A. Rogers, W. Hwang, and L. Scott. 2004. The effects of work breaks on staff nurse performance. Journal of Nursing Administration 34, 11 (2004), 512-9.

[18] A. Rogers, W. Hwang, L. Scott, L. Aiken, and D. Dinges. 2004. The working hours of hospital staff nurses and patient safety. Health Affairs 23, 4 (2004), 202-12.

[19] J. Zimmerman, J. Forlizzi, and S. Evenson. 2007. Research Through Design As a Method for Interaction Design Research in HCI. In Proc. of CHI '07. ACM, 493-502. 